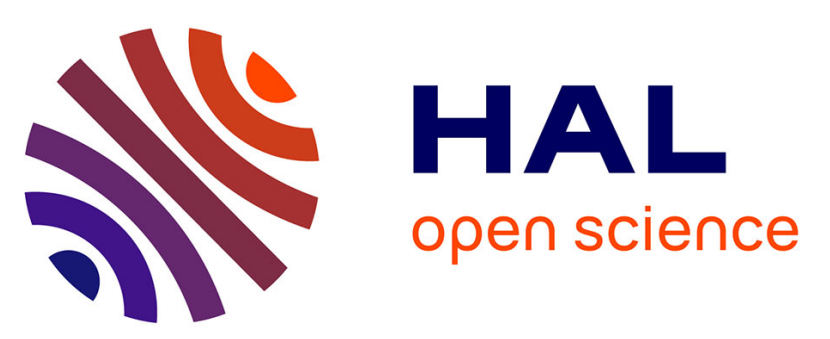

\title{
Measurement of the optical absorption of bulk silicon at cryogenic temperature and the implication for the Einstein Telescope
}

J Degallaix, J Komma, Danièle Forest, G Hofmann, M Granata, D Heinert, C Schwarz, R Nawrodt, L Pinard, Christophe Michel, et al.

\section{To cite this version:}

J Degallaix, J Komma, Danièle Forest, G Hofmann, M Granata, et al.. Measurement of the optical absorption of bulk silicon at cryogenic temperature and the implication for the Einstein Telescope. Classical and Quantum Gravity, 2014, 31 (18), 10.1088/0264-9381/31/18/185010 . in2p3-01357342

HAL Id: in2p3-01357342

https://hal.in2p3.fr/in2p3-01357342

Submitted on 29 Aug 2016

HAL is a multi-disciplinary open access archive for the deposit and dissemination of scientific research documents, whether they are published or not. The documents may come from teaching and research institutions in France or abroad, or from public or private research centers.
L'archive ouverte pluridisciplinaire HAL, est destinée au dépôt et à la diffusion de documents scientifiques de niveau recherche, publiés ou non, émanant des établissements d'enseignement et de recherche français ou étrangers, des laboratoires publics ou privés. 
Measurement of the optical absorption of bulk silicon at cryogenic temperature and the implication for the Einstein Telescope

This content has been downloaded from IOPscience. Please scroll down to see the full text.

2014 Class. Quantum Grav. 31185010

(http://iopscience.iop.org/0264-9381/31/18/185010)

View the table of contents for this issue, or go to the journal homepage for more

Download details:

IP Address: 134.214.188.172

This content was downloaded on 09/09/2014 at 09:27

Please note that terms and conditions apply. 


\title{
Measurement of the optical absorption of bulk silicon at cryogenic temperature and the implication for the Einstein Telescope
}

\author{
J Degallaix ${ }^{1}$, J Komma ${ }^{2}$, D Forest ${ }^{1}$, G Hofmann ${ }^{2}$, M Granata ${ }^{1}$, \\ D Heinert ${ }^{2}$, C Schwarz ${ }^{2}$, R Nawrodt ${ }^{2}$, L Pinard ${ }^{1}$, C Michel ${ }^{1}$, \\ R Flaminio ${ }^{1}$ and $\mathrm{G}$ Cagnoli $^{1}$ \\ ${ }^{1}$ Laboratoire des Matériaux Avancés, IN2P3/CNRS, Université de Lyon, 7 Avenue \\ Pierre de Coubertin, F-69100 Villeurbanne, France \\ ${ }^{2}$ Friedrich-Schiller-Universität Jena, Institut für Festkörperphysik, Helmholtzweg 5, \\ D-07743 Jena, Germany \\ E-mail: j.degallaix@lma.in2p3.fr
}

Received 23 May 2014, revised 10 July 2014

Accepted for publication 17 July 2014

Published 28 August 2014

\begin{abstract}
We report in this article on the measurement of the optical absorption of moderately doped crystalline silicon samples at $1550 \mathrm{~nm}$, which is a candidate material for the main optics of the low temperature interferometer of the Einstein Telescope (ET). We observe a nearly constant absorption from room temperature down to cryogenic temperatures for two silicon samples presenting an optical absorption of $0.029 \mathrm{~cm}^{-1}$ and $780 \mathrm{ppm} \mathrm{cm}^{-1}$, both crystals doped with boron. This is in contradiction to what was assumed previously-a negligible optical absorption at low temperature due to the carrier freezeout. As the main consequence, if the silicon intrinsic absorption can not be lowered, the cross section of the mirror suspension of the ET must be increased to be able to carry away the excess heat generated by the partially absorbed laser beam during the operation of the interferometer.
\end{abstract}

Keywords: silicon, Einstein Telescope, optical absorption PACS numbers: 78.40.Fy, 04.80.Nn, 95.55Ym

(Some figures may appear in colour only in the online journal)

\section{Introduction}

The Einstein Telescope (ET) is a European project aimed at directly detecting gravitational waves from astrophysical sources on a daily basis [1]. This detector is the planned successor 
of the Advanced LIGO and Advanced Virgo interferometers currently in their installation phase and early commissioning [2]. Those advanced detectors are expected to achieve the first direct detection of a gravitational wave within the next five years and hence open a new window to study the universe, complimentary to our current vision based on the electromagnetic spectrum.

To greatly increase the detection rate in the following decade, the ET has been designed to be ten times more sensitive than the advanced detectors [1], probing a volume of space a thousand times larger. To achieve this goal, this observatory will be composed of two complementary interferometers sharing a common underground infrastructure. One high frequency interferometer (ET-HF) will be at room temperature with fused silica mirrors similar to the advanced detectors. The low frequency interferometer (ET-LF) will be more innovative using silicon mirrors operating at cryogenic temperatures.

Since the cavity input test masses made of silicon will be used in transmission, the optical absorption is an essential parameter which determines the amount of heat generated in the optics, which is equal to the amount of heat to be removed through the suspensions in order to keep the mirrors at their operating cryogenic temperature [3]. Additionally it seems unlikely that high purity silicon (or even intrinsic silicon having no significant doping/impurities) will be available in large enough pieces being suited for ET. Thus the focus of this work lies on moderately doped silicon that can be provided by Czochralski growth of single crystals; by 'moderately' we mean samples with resistivities ranging from few $\Omega \mathrm{cm}$ to few $\mathrm{k} \Omega \mathrm{cm}$.

Contrary to the low temperature regime, the optical absorption of bulk silicon at room temperature and in the near infrared is well understood and characterized $[4,5]$. The optical absorption is proportional to the amount of free carriers (the proportionality coefficient been simply called the free carrier coefficient). At room temperature the concentration of the free carriers is itself equal to the concentration of dopant impurities as every atom of dopant is ionized and gives either one electron in the conduction band (n-type) or one hole in the valence band (p-type). In the case of moderately doped samples all dopants are ionized at room temperature. Thus, the concentration of free carriers is equal to the concentration of doping elements.

At cryogenic temperatures, below $40 \mathrm{~K}$, the thermal energy is not enough to ionize the impurities and hence the amount of free carrier drops and rapidly becomes very small. It was reasonably expected that the optical absorption of such samples also drops rapidly proportionally to the free carrier concentration. However, we demonstrate in this paper that although the concentration of free carriers drops as expected the optical absorption nearly stays constant throughout a wide temperature range from $300 \mathrm{~K}$ down to $5 \mathrm{~K}$.

The measurements are done using two independent techniques based on photodeflection and calorimetry. Using a third technique (based on spectrophotometric measurement) Spitzer and Fan [6] already showed no change in absorption for highly doped silicon wafers from room temperature down to $5 \mathrm{~K}$.

This article is organised as follows: first the two techniques used to measure the optical absorption are explained; second, the experimental setup as well as the silicon samples tested are described and third the experimental results are presented. Finally, the consequences of theses measurements for the ET are derived.

\section{Measurement techniques and experimental setups}

In this section, the two experimental techniques used to derive the optical absorption of bulk silicon are presented. The two techniques applied in this paper-the mirage effect and the 
heating of the sample by light - are sensitive enough to measure the absorption of moderately doped bulk silicon, the expected material of the ET cryogenic mirror. For samples with higher optical absorption (larger than $0.1 \mathrm{~cm}^{-1}$ ), a direct measurement of the transmission is enough to derive the absorption [6].

After the measurement principles are summarized, the experimental setups as well as the silicon samples which have been measured are explained in detail.

\subsection{The photothermal deflection technique}

The photodeflection technique based on the mirage effect is one of the most sensitive methods to measure low optical absorption in materials [7]. Using this very same technique, it has already been shown that pure silicon can present an absorption as low as $5 \mathrm{pm} \mathrm{cm}^{-1}$ at room temperature [5].

The typical setup of a photodeflection bench is composed of two lasers: one high power laser called the pump laser and one low power laser, the probe laser. The pump laser is focused on the material and so is partially absorbed within the sample. The absorbed optical power, converted to heat creates a gradient of temperature. The gradient of temperature induces a gradient of refractive index via the thermo-optic coefficient. The probe beam is aligned to intersect the pump beam in the sample and is then deflected by the gradient of refractive index. The deviation of the probe beam is read out by a quadrant photodetector and is directly proportional to the amount of power absorbed in the sample and hence to the optical absorption.

In order to increase the signal-to-noise-ratio of the deviation method, the pump beam is intensity modulated and the deviation is then detected by a lock-in amplifier. The calibration which links the deviation amplitude to the optical absorption is performed with a sample with a high optical absorption that was directly measured by a spectrophotometer.

\subsection{Calorimetric measurement}

A calorimetric measurement technique determines the temperature increase of a sample under illumination by a laser of wavelength under study [8]. The temperature increase is directly linked to the optical absorption if the heat capacity of the setup as well as all thermal losses are known. Calorimetric techniques using lasers as a light source are routine techniques allowing the measurement of absorptions below $1 \mathrm{ppm} \mathrm{cm}^{-1}$ for high purity optical elements or fiber materials [9, 10].

A laser beam is passed through a sample and a temperature sensor is measuring the increase in temperature under illumination. Care must be taken to avoid any scattered light to hit the sensor directly. This method probes the integral optical absorption of the sample-both possible surface absorption as well as intrinsic bulk absorption. By changing the length of the sample it is possible to disentangle these effects.

In order to reduce extrinsic surface absorption effects great care was taken to clean the samples. A sophisticated cleaning technique available from the semiconductor industry was used and described in section 3.1.

The calorimetric technique becomes especially very sensitive at cryogenic temperatures as the heat capacity of the samples rapidly drops below $50 \mathrm{~K}$ following Debye's law $\left(\propto T^{3}\right)$.

\subsection{Description of the setup at $L M A$}

The photodeflection bench used for the measurement presented in this article is composed of a $30 \mathrm{~W}$ pump laser at a wavelength of $1550 \mathrm{~nm}$ which is the same wavelength planned for ET 


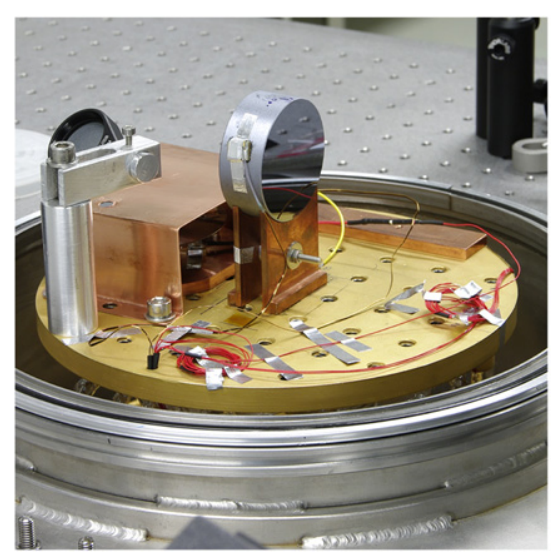

(a)

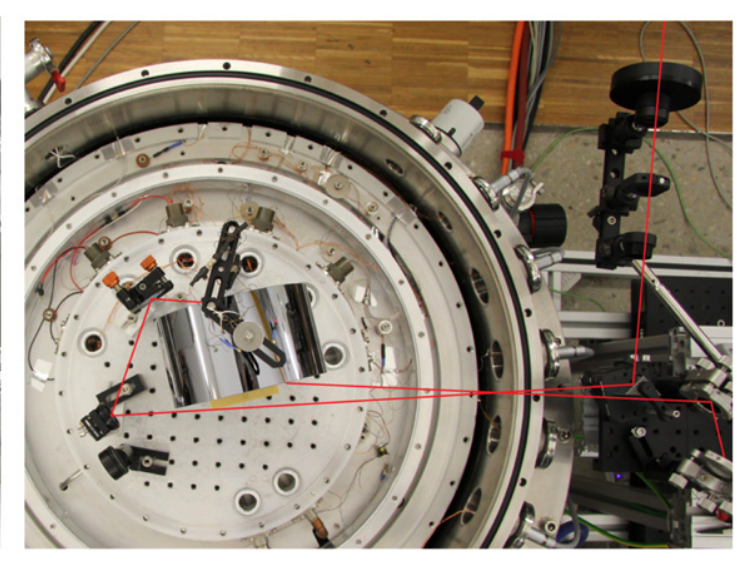

(b)

Figure 1. Photography of the silicon samples in the cryostats at LMA (a) and at the FSU Jena (b). See the text for additional details.

and a $1 \mathrm{~mW}$ probe laser at $1310 \mathrm{~nm}$. The wavelength of the probe beam is selected to be in the transparency region of the silicon and close enough to $1550 \mathrm{~nm}$ to ensure a similar thermorefractive coefficient [11]. The pump beam is focused in the silicon sample with a measured waist radius of $0.7 \mathrm{~mm}$ (measured with the knife edge technique at the position of the sample) resulting in power densities of maximum $4 \mathrm{~kW} \mathrm{~cm}^{-2}$.

The tested silicon sample is located in a continuous flow cryostat linked to a liquid helium reservoir. The cooling from room temperature to $10 \mathrm{~K}$ is achieved within 24 hours with the sample mounted to a $20 \mathrm{~cm}$ diameter cryogenic breadboard via a holder made of copper. A calibrated temperature sensor (Cernox-1070-BO) is directly attached to the sample to continuously record the temperature of the silicon substrate, as shown in figure 1(a). Several optical glass windows with a diameter of 2 inches have been installed to ensure the correct injection and extraction of the pump and probe laser beams. Further details on the cryostat performance can be found in a previous article [12].

For the LMA cryogenic run described in this article, the tested silicon sample has a diameter of two inches and is $20 \mathrm{~mm}$ long. The sample is doped with boron atoms, giving an averaged resistivity of $2.4 \Omega \mathrm{cm}$ (concentration of boron atoms of $5.7 \times 10^{15} \mathrm{~cm}^{-3}$ ). The optical absorption at room temperature due to the free carriers was measured to be $0.029 \mathrm{~cm}^{-1}$ at $1550 \mathrm{~nm}$.

\subsection{Description of the setup at the FSU Jena}

The setup at the FSU Jena consists of a $5 \mathrm{~W} \mathrm{CW} 1550 \mathrm{~nm}$ fiber laser system (NKT Photonics Koheras Adjustic seed laser and Boostik HPA amplifier) and a custom-made bath cryostat [13] for measurements between room temperature and $5 \mathrm{~K}$. The laser beam has a measured waist radius of $0.9 \mathrm{~mm}$ inside the sample resulting in a power density of $0.4 \mathrm{~kW} \mathrm{~cm}^{-2}$. The sample under investigation is a float zone $100 \Omega \mathrm{cm}$ silicon single crystal having a diameter of $150 \mathrm{~mm}$ and a thickness of $86 \mathrm{~mm}$ as shown in figure 1(a). The concentration of boron atoms is about $1 \times 10^{14} \mathrm{~cm}^{-3}$.

A temperature sensor (Cernox CX1070-SD) is directly attached to the sample by means of varnish (VGE 7031). Additionally, an electrical heater is attached to the sample for calibration purposes. Using such a heater avoids the need of a careful treatment of heat losses 
in the setup. Applying an electric heating power of $1 \mathrm{~mW}$ results in the same heating as is caused by $1 \mathrm{~mW}$ of absorbed optical power-independent of all heat losses through the mount, wires or even radiation. These heat losses are kept small by avoiding too much heating of the sample as all heat losses are driven by temperature differences. Typically, no temperature difference larger than $100 \mathrm{mK}$ was used in the experiments.

The sample is mounted inside the cryostat and a weak thermal link is established between the sample and the cold plate. For initial cooling He gas can be applied to the probe chamber allowing an efficient and fast cooling of the sample. The laser beam is aligned in a way that the sample is illuminated under the Brewster angle to avoid unwanted reflections. By means of a set of mirrors the laser beam is then aligned in a way that it leaves the cryostat through the same window as it enters. This technique is needed as the cryostat is only equipped with one window.

\section{Experimental investigations}

The optical absorption measured at low temperature is summarized in the following sections and compared to the values found at room temperature.

\subsection{Investigation of a possible surface absorption}

Optical absorption measurements done before on a monolithic cavity [14] reported a high surface absorption. Also, the calorimetric measurements will be extremely sensitive to such absorptions - either being caused by intrinsic surface effects or simply dirt on the surface. Thus, a special run was initially carried on to check the cleaning procedure used for the samples. All samples have been cleaned using a hot mixture of sulfuric acid $(96 \%)$ and hydrogen peroxide $(35 \%)$. This mixture does not attack silicon but efficiently removes all organic contamination from the surface. After this cleaning process all samples are given a methanol rinse using ultra pure solvents.

As the surface is the potential origin of unwanted absorption thin wafers have been used for testing surface effects. These wafers are made from high purity material (typically between $10-100 \mathrm{k} \Omega \mathrm{cm}$ ) whose intrinsic absorption is expected to be below $4 \mathrm{ppm} \mathrm{cm}^{-1}$ [5]. Such samples have been used to refine the cleaning procedure. A minimum average absorption over at least ten subsequent runs of $(12 \pm 9) \mathrm{ppm}$ has been observed. As all absorption values reported in this paper are well above $100 \mathrm{ppm} \mathrm{cm}^{-1}$ any influence of the surface to our measurements can be excluded. Additionally, these results make the hypothesis of potential intrinsic strong surface absorptions in silicon very unlikely [14].

\subsection{Freeze-out of free carriers}

As the optical absorption of silicon is dominated by free carrier absorption at room temperature, it is an interesting step to investigate the change of free carriers during cooling. In order to do so, a moderately doped silicon wafer sample (1-10 $\Omega \mathrm{cm}$, p-type boron-doped) was contacted in a four point geometry as shown in figure 2. Four aluminium pads were evaporated onto the surface of the sample forming electronic contacts. Low contact resistivity Ohmic contacts have been achived by a careful annealing of the sample. The four contact pads have been attached to wires of a cryogenic probe station by means of indium. The two outer contacts have then be used to feed a constant current through the sample while the inner contacts have been used to measure the voltage created by the current. An Agilent B2901A SMU has been used to apply the current and measure the voltage. The finite geometry of the 


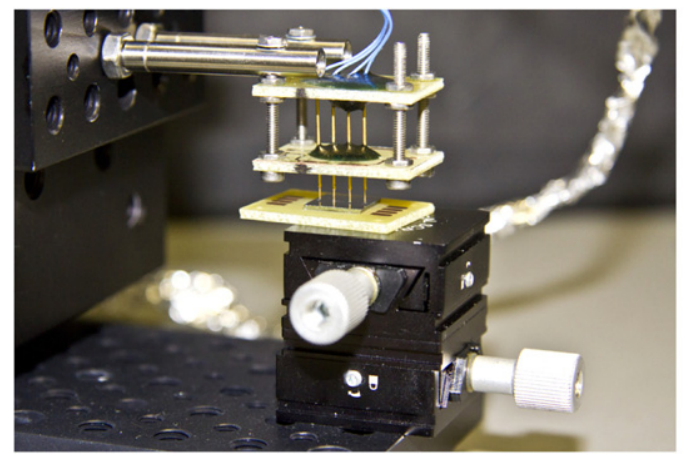

(a)

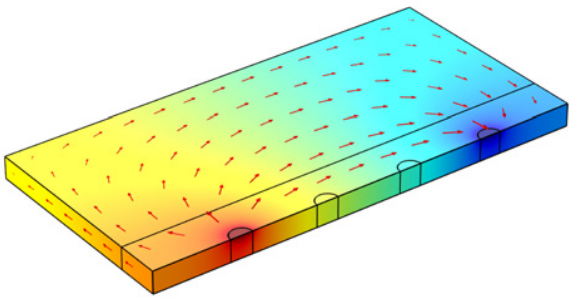

(b)

Figure 2. (a) 4-probe-setup for measuring the resistance of the silicon sample. (b) Distribution of the electrical potential within the sample to include boundary effects of the finite sized sample using COMSOL.

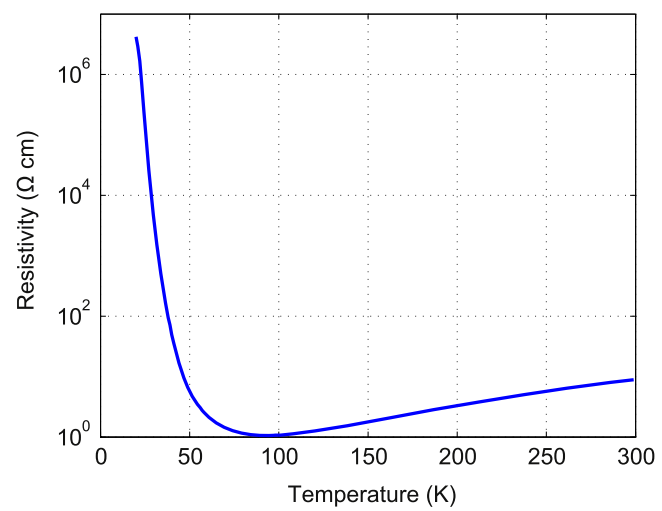

Figure 3. Measured values of the resistivity of the silicon sample $(1-10 \Omega \mathrm{cm}$, boron doped) under investigation in a wide temperature range from $5 \mathrm{~K}$ to $300 \mathrm{~K}$.

sample in use (typically $10 \mathrm{~mm} \times 10 \mathrm{~mm}$ ) has been taken into account numerically by means of a FEA using COMSOL.

Figure 3 shows the results obtained from a voltage-current-measurement over a wide range of temperatures from $5 \mathrm{~K}$ to $300 \mathrm{~K}$. It can be seen that the resistivity starts at around $8 \Omega \mathrm{cm}$ at room temperature, decreases to about $1 \Omega \mathrm{cm}$ at $80 \mathrm{~K}$ and then steeply rises to more than $10 \mathrm{M} \Omega \mathrm{cm}$ at cryogenic temperatures. Here, the measurement is limited by the finite input resistance of the pre-amplifier in use.

The resistivity $\rho$ of a semiconductor is given by [15]

$$
\rho=\frac{1}{-e n \mu_{n}+e p \mu_{p}}
$$

with the mobility of the electrons $\mu_{n}$ and the holes $\mu_{p}$ and the density of electrons $n$ and holes $p$. In the case studied here holes are mainly carrying the current (as the sample is p-doped). Thus, the concentration of electrons can be approximated to be zero. The observed behaviour of the resistivity by changing the temperature cannot be described by the temperature dependence of the mobility $\mu_{p}$ (see e. g. [16]) thus the change in hole concentration is the only 


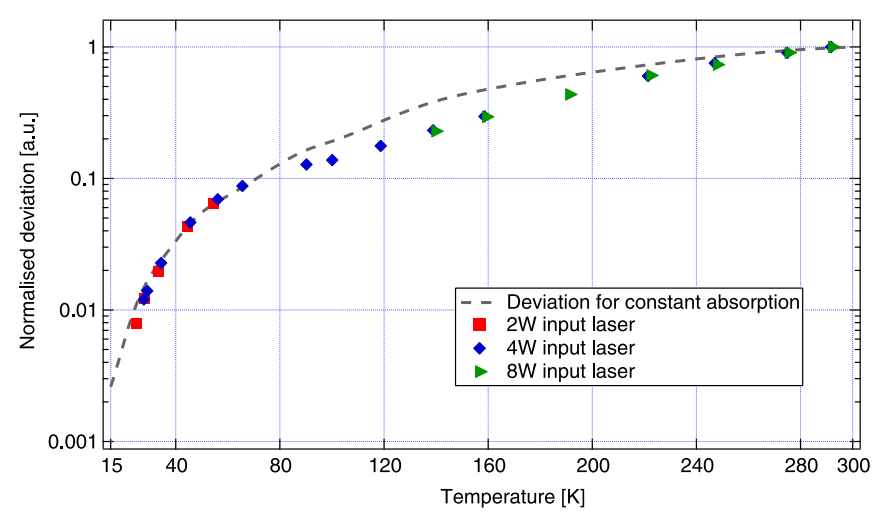

Figure 4. Normalized deviation of the probe beam as a function of the temperature for the photodeflection bench. The dashed curve represents the expected theoretical deviation if the optical absorption is constant with the temperature.

explanation for the measured trend presented in figure 3. The concentration of free carriers is therefore strongly reduced by cooling below $50 \mathrm{~K}$.

\subsection{Bulk absorption measurement at LMA}

The photodeflection technique presented in section 2.1 has been very successful at room temperature however it remains an indirect measurement where physical parameters of the substrate play an important role. For example, the deviation of the probe beam is different for two samples presenting the same optical absorption if they have different thermal conductivities or thermo-refractive coefficient.

The same phenomenon happens when the temperature of the silicon sample changes. The measured deviation will change not only because of a possible change in the absorption but also since the specific heat, the thermal conductivity and the thermo-refractive coefficient depend on temperature. We can already predict that the deviation signal for a constant optical absorption will decrease with temperature as the thermooptic coefficient decreases and the thermal conductivity increases for silicon substrate.

More precisely, for silicon the thermo-refractive coefficient will decrease by a factor 100 from room temperature to $20 \mathrm{~K}$ [17], hence reducing the gradient of refractive index responsible for the deviation of the probe beam. That would be the major effect, the change in thermal conductivity [18] and specific heat having a smaller effect [19]. That statement is valid for our setup and dependent on the modulation frequency of the pump beam as well as the size of the pump laser beam. So we already expect that the deviation signal will decrease by a factor 100 between room temperature and cryogenic temperature if the absorption is constant. So, for this reason, we tested a sample with relatively high optical absorption $\left(0.029 \mathrm{~cm}^{-1}\right)$ in order to have large enough deviation signal from $300 \mathrm{~K}$ to $10 \mathrm{~K}$ (the SNR of the deviation signal is around $2 \times 10^{3}$ at room temperature).

The theoretically predicted deviation signal as a function of temperature is shown as the dashed line in figure 4, and assuming a constant absorption for the silicon sample. On that figure, the deviation is normalized to be 1 at room temperature. The measured deviation, also normalized to the value at room temperature, is also added to the figure.

The deviation has been measured at different pump powers to rule out nonlinear effects. Also near room temperature, high pump power is used to generate a noticeable rise in the 
(a)

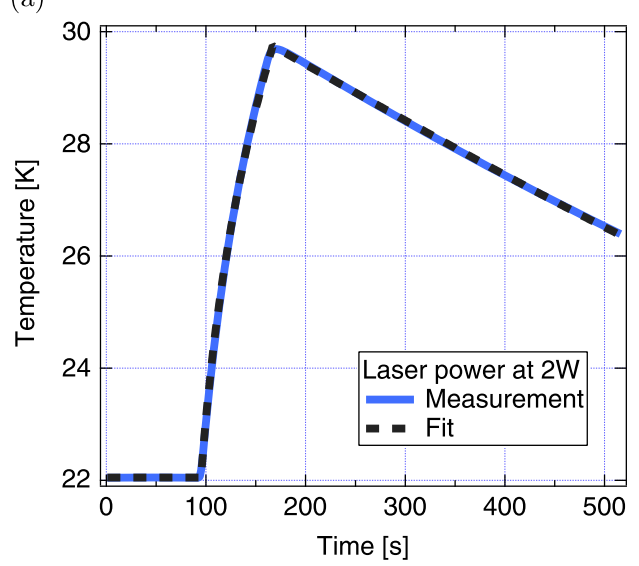

(b)

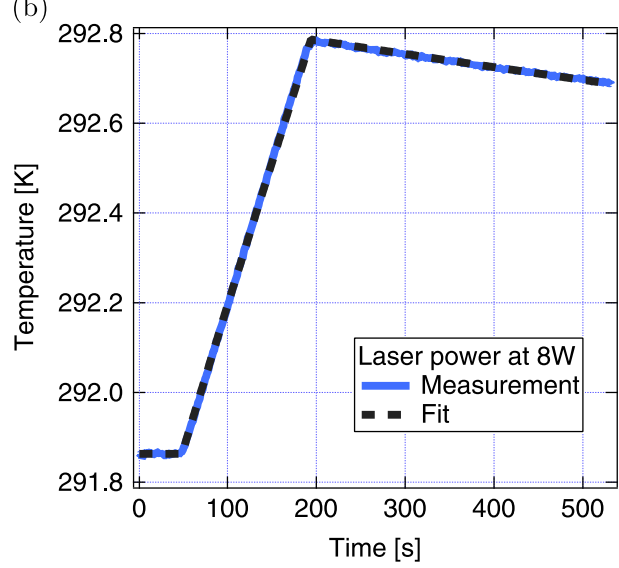

Figure 5. Example of a temperature rise at cryogenic temperature (a) and room temperature (b) when the pump laser is switched on for few minutes. For the left plot, the pump (heating) laser is switched on at $94 \mathrm{~s}$ and switched off at $164 \mathrm{~s}$ and for the right plot the times are respectively $48 \mathrm{~s}$ and $193 \mathrm{~s}$. The laser power is set to $2 \mathrm{~W}$ at cryogenic temperature but to $8 \mathrm{~W}$ at room temperature in order to create a noticeable temperature rise.

temperature of the sample in order to still derive the absorption also from calorimetric measurement. The deviation measurement were also normalized with the pump power to compare all the data points to the same theoretical curve.

At low temperature, below $50 \mathrm{~K}$, we can notice from graph 4 that the measured deviation matches the theoretical deviation from constant absorption within $20 \%$. This result indicates that the optical absorption is the same at room temperature and low temperature for bulk silicon.

At the same time as the measurement is done with the photodeflection technique, the temperature of the sample is monitored. In that way, the heating due to the pump laser beam is recorded. Such a plot of the temperature evolution is shown in the left part of the figure 5 .

To derive the heating power from the temperature rise a fit is performed. The fitting function is given by solving the heat equation in the simple case of assuming an isothermal behaviour of the sample. This assumption is valid for silicon since the thermal conductivity is very high in the temperature range under investigation (with the thermal conductivity being always larger than $150 \mathrm{~W}\left(\mathrm{~m} \mathrm{~K}^{-1}\right)$. Such fits are presented in figure 5 . The fit is remarkably good, considering the simplicity of the model and that only two parameters are fitted: the absorbed optical power and the heat lost by conduction through the mount of the sample.

From the fit, at low temperature $(22 \mathrm{~K})$, the heating power absorbed by the sample is found to be $0.11 \mathrm{~W}$ for an input laser of $2 \mathrm{~W}$, giving an optical absorption of $0.030 \mathrm{~cm}^{-1}$. At room temperature we found an absorbed power of $0.44 \mathrm{~W}$ for an input power of $8 \mathrm{~W}$ which gives the same absorption as at low temperature. The uncertainty on such measurement is within five percent coming from the uncertainty on the specific heat (especially at low temperature) and on the measured laser power. The measured absorption at room temperature derived from the calorimetric measurement is within $2 \%$ of the value found by spectroscopic measurement.

The results from the calorimetric measurement suggest that no decrease (nor increase) in the optical absorption of silicon should be expected by cooling the substrate from room 

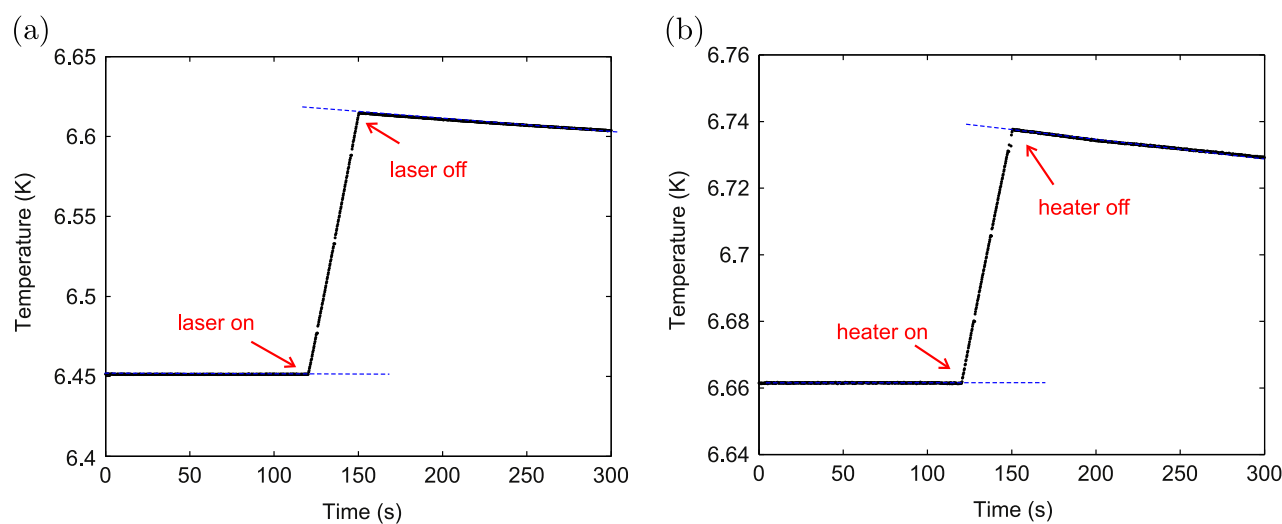

Figure 6. Example for a calorimetric measurement run. The sample is heated by a laser (a) and by an electrical heater (b). The electric heating is used for calibration purposes. The laser power was $0.26 \mathrm{~W}$ and the electrical heater power $0.73 \mathrm{~mW}$. In both curves the heating time was $30 \mathrm{~s}$.

temperature to $20 \mathrm{~K}$. That is the same conclusion as the measurement based on the photodeflection technique.

\subsection{Bulk absorption measurement at the FSU Jena}

Figure 6 represents a typical set of measured values at cryogenic temperatures. The temperature of the sample is first stabilized and recorded for a given time of a few minutes. Then the laser illuminates the sample for a certain time interval with a certain laser power. Illumination time and laser power are adjusted in a way that the resulting temperature increase can be easily measured and is not too large. Under this assumption the thermal properties of the sample can be assumed to be constant. Typical temperature differences of a few $10 \mathrm{mK}$ are used. After the thermal equilibrium is reached again an electrical heater is used to measure the temperature increase for a given electrical heating power. The power is roughly adjusted to give a similar temperature increase during this period. This method allows to eliminate unknown parasitic heat fluxes to the surroundings.

Heating the sample over a time $\Delta t$ with a power $P$ results in a temperature increase $\Delta T$ of

$$
P \Delta t=C \Delta T
$$

with $C$ being the net heat capacity of the sample. This is independent whether the power is delivered by a laser or an electrical heater. Using the subscript $\mathrm{L}$ for laser and $\mathrm{H}$ for heater allows the substitution of the unknown heat capacity $C$. The absorbed laser power $P_{L}$ is then given by

$$
P_{L}=\frac{\Delta T_{L}}{\Delta T_{H}} \frac{\Delta t_{H}}{\Delta t_{L}} P_{H}
$$

All input parameters can be easily measured assuming that the heat capacity is the same during laser illumination and electrical heating. Under the experimental constraints used in this study this is always fulfilled.

The absorbed laser power $P_{L}$ is linked to the illuminating laser power $P_{0}$, the absorption coefficient $\alpha$ and the optical path of the laser beam inside the sample $L$ by means of 


$$
P_{L}=P_{0} e^{-\alpha L}
$$

Equations (3) and (4) are used to determine the optical absorption $\alpha$. The measurement of the $150 \mathrm{~mm}$ bulk sample shows the same absorption at room temperature and at $6 \mathrm{~K}$, which is $780 \mathrm{ppm} / \mathrm{cm}$. An independent cross-check using the photodeflection technique at LMA revealed a similar value for the room temperature value. Thus, also this second sample exhibiting a reduced concentration of dopants shows similar absorption values at $300 \mathrm{~K}$ and $6 \mathrm{~K}$. This independently confirms the findings on the sample previously discussed in section 3.3 and suggests that the obtained behaviour might be inherent to silicon.

The measurement has been repeated at least ten times at the different temperatures resulting in a typical statistical fluctuation of $10 \%$ at room temperature and $1 \%$ at cryogenic temperatures. The higher accuracy at low temperatures is based on the larger temperature difference that needs to be measured due to the smaller heat capacity. Further including the inaccuracy of the laser power sensors as well as of the heater power results in a total relative error of $20 \%$ at room temperature and $10 \%$ at cryogenic temperatures.

\section{Consequences for ET}

To derive the numbers presented in this section, several parameters of the ET have to be taken into account. Relevant parameters are taken from the ET conceptual design study [20] and are presented below.

The circulating power in the core interferometer is expected to be $65 \mathrm{~W}$ (measured on the recycling mirror) and the power circulating in the arm cavity is $18 \mathrm{~kW}$, that is also the power incident to the high reflectivity coating of the input mirror.

The silicon mirror has a cylindrical shape with a diameter of $50 \mathrm{~cm}$ and a thickness of $46 \mathrm{~cm}$. The operating temperature is assumed to be $10 \mathrm{~K}$ and at the time of writing the design study the absorbed power was estimated to be $20 \mathrm{~mW}$.

Potentially, Czochralski grown silicon having a resistivity between $500 \Omega \mathrm{cm}$ and $1000 \Omega \mathrm{cm}$ will be available in large enough pieces for optics that can be used in ET-LF [21]. Here, silicon single crystals with a diameter of $50 \mathrm{~cm}$ and thicknesses of about $50 \mathrm{~cm}$ are needed. Higher purities (and hence nominally low absorptions [5]) can be obtained by means of the float zone technique-however, it is not expected that it can be produced in such large pieces within the next years as currently a technical limitation in size to about $200-250 \mathrm{~mm}$ exists.

As we have shown in this article, the optical absorption at cryogenic temperatures is roughly the same as at room temperature. Thus, silicon of the purity discussed here will have an absorption of about $300 \mathrm{ppm} \mathrm{cm}^{-1}$ throughout the whole temperature range from $300 \mathrm{~K}$ down to $5 \mathrm{~K}$ [5]. This assumption is already conservative accounting for a safety margin in the following estimates.

The most crucial mirror affected by optical absorption is the input test mass of the arm cavities of ET-LF. Here, a laser beam of approximately $65 \mathrm{~W}$ will pass through the whole material made of silicon. The total absorption is then given by

$$
P_{\mathrm{abs}}=\alpha \times L \times P_{0}
$$

with the absorption coefficient $\alpha$, the length of the substrate $L$ and the laser power $P_{0}$. Using the above values, the total absorbed power within the silicon material is around $1 \mathrm{~W}$. This heat needs to be removed by the suspension elements being planned to be fabricated by highly conductive silicon material. 


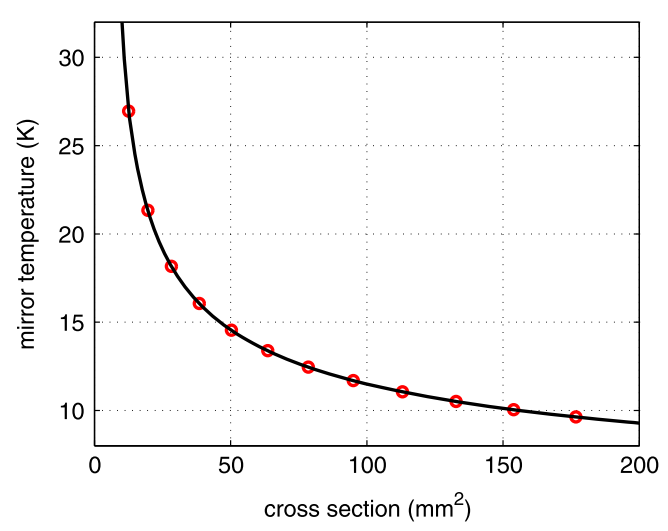

Figure 7. Equilibrium temperatures in the input test mass assuming a realistic optical absorption as well as a temperature dependent thermal conductivity for silicon. The temperature at the cold end of the fiber was assumed to be $4 \mathrm{~K}$, the lengths of the fiber is set to $1 \mathrm{~m}$.

Assuming a suspension length of $1 \mathrm{~m}$, the equilibrium temperature of the mirror was calculated using an FEA model in COMSOL. Here, the temperature dependence of the thermal conductivity of silicon as well as its size dependence was accounted for. As a simplification a single suspension fiber was assumed - a simple scaling using the real number and length of the fiber can be done easily. Figure 7 summarizes the results.

It can be directly seen that the expected operational temperature of $10 \mathrm{~K}$ at the test mass for ET-LF is only possible with a total cross section of more than $150 \mathrm{~mm}^{2}$. This might result in a very stiff suspension showing bad thermal noise performance [22]. Thus, a higher operational temperature for the ET-LF mirrors is preferred which is also compatible with the tolerable mirror thermal noise level. At around $20 \mathrm{~K}$ a total cross section of about $25 \mathrm{~mm}^{2}$ is needed which can be provided by four suspension wires or ribbons.

A further reduction of the temperature of the cold end does not give any benefits for the heat extraction as the thermal conductivity of silicon is small between $2 \mathrm{~K}$ and $4 \mathrm{~K}$. This does not lead to a better cooling performance. However, using LHe in the superfluid state might result in a dramatic reduction of mechanical disturbances and could be appealing for the overall performance.

In parallel to the development of suitable cryogenic suspension, research on silicon substrates will continue with the aim to find the silicon with the lowest possible absorption at low temperature and available in sizes required for ET.

The larger thermal absorption in the bulk material relaxes the constraints on the coating absorption significantly. Considering the laser power of $18 \mathrm{~kW}$ in the interferometer arm cavities and assuming that the coating is allowed to absorb a tenth of power absorbed in the substrate (i.e. $0.1 \mathrm{~W})$, a maximum coating absorption of $5 \mathrm{ppm}(\approx 0.1 \mathrm{~W} / 18 \mathrm{~kW})$ can be tolerated. A higher tolerable coating absorption has some important consequences since it opens the possibility to coating technology different from the usual alternative layers of fused silica/tantala [23]. More specifically, crystalline coating [24] or hafnia layers [25] to replace tantala may be acceptable. 


\section{Conclusion}

Based on independent measurements on two silicon samples, we have shown that no significant modification in the optical absorption at $1550 \mathrm{~nm}$ of bulk crystalline silicon must be expected by cooling the substrate from room temperature to cryogenic temperature. This result is in contradiction to what was assumed for the cryogenic silicon mirrors of the ET, since it was thought that the free carrier freezeout happening at low temperature will guarantee a negligible optical absorption. Further, no significant surface absorption has been observed during our measurement.

Our conclusion implies that a relatively large amount of heat will have to be extracted from the cryogenic mirrors in order to keep the desired temperature of the interferometer if the current silicon absorption cannot be lowered. As a result, in the current design of the suspension the cross section of the fiber holding the mirrors must be significantly increased with the risk of increasing the thermal noise. Further detailed studies are needed to optimise the shape, material selection as well as the optimum operational temperature.

If the heat absorbed within the mirror substrate is relatively high, the constraints on the other potential sources of heat occurring in the mirrors can be relaxed. In particular, the coating absorption could be as high as $5 \mathrm{ppm}$ and more heat from radiation could be tolerated from the room temperature vacuum enclosure.

According to our tests for all practical purposes the absorption can be considered constant between room temperature and low temperature. So, in order to select the suitable silicon quality for the ET mirrors, a measuring campaign for the optical absorption at room temperature is likely to be sufficient without the need of expensive cryogenic runs. Of course, once a particular quality of silicon has been selected, a confirmation of the absorption at low temperature will still be required.

The measured absorption at low temperature is so far unexplained by any theory known by the authors. Research to understand why the absorption is only marginally dependent on the temperature is ongoing.

\section{Acknowledgements}

The authors from LMA gratefully acknowledge the support of the European Gravitational Observatory (EGO) and the LABEX Lyon Institute of Origins (ANR-10-LABX-0066) of the Universit de Lyon for its financial support within the program Investissements dAvenir (ANR-11-IDEX-0007) of the French government operated by the National Research Agency (ANR). The support of the German Science Foundation DFG is acknowledged under contract SFB TR7. The EU supports this activity under the APPEC grant ET R\&D.

\section{References}

[1] Punturo M et al 2010 The Einstein telescope: a third-generation gravitational wave observatory Class. Quantum Grav. 27194002

[2] Losurdo G 2012 Ground-based gravitational wave interferometric detectors of the first and second generation: an overview Class. Quantum Grav. 29124005

[3] Tomaru T et al 2002 Maximum heat transfer along a sapphire suspension fiber for a cryogenic interferometric gravitational wave detector Phys. Lett. A 301 215-9

[4] Soref R A and Bennett B R 1987 Electrooptical effects in silicon IEEE J. Quantum Electron. 23 123-9 
[5] Degallaix J et al 2013 Bulk optical absorption of high resistivity silicon at $1550 \mathrm{~nm}$ Opt. Lett. 38 2047-9

[6] Spitzer W and Fan H Y 1957 Infrared absorption in n-type silicon Phys. Rev. 108 268-71

[7] Jackson W B, Amer N M, Boccara A C and Fournier D 1981 Photothermal deflection spectroscopy and detection Appl. Opt. 20 1333-44

[8] Tomaru T et al 2001 Cryogenic measurement of the optical absorption coefficient in sapphire crystals at $1.064 \mu \mathrm{m}$ for the large-scale cryogenic gravitational wave telescope Phys. Lett. A 283 80-84

[9] Willamowski U, Ristau D and Welsch E 1998 Measuring the Absolute Absorptance of Optical Laser Components Appl. Opt. 37 8362-70

[10] Stone F T, Gardner W B and Lovelace C R 1978 Calorimetric measurement of absorption and scattering losses in optical fibers Opt. Lett. 2 48-50

[11] Frey B J, Leviton D B and Madison T J 2006 Temperature-dependent refractive index of silicon and germanium Astron. Telesc. Instrum. Int. Soc. Opt. Photonics 62732J

[12] Degallaix J et al 2012 The new cryogenic facility at LMA J. Phys.: Conf. Ser. 363012008

[13] Nawrodt R, Zimmer A, Nietzsche S, Thürk M, Vodel W and Seidel P 2006 A new apparatus for mechanical Q-factor measurements between 5 and $300 \mathrm{~K}$ Cryogenics 46 718-23

[14] Khalaidovski A, Steinlechner J and Schnabel R 2013 Indication for dominating surface absorption in crystalline silicon test masses at $1550 \mathrm{~nm}$ Class. Quantum Grav. 30165001

[15] Grundmann M 2010 The Physics of Semiconductors: An Introduction Including Nanophysics and Applications (Graduate Texts in Physics) (Berlin: Springer)

[16] Jacoboni C, Canali C, Ottaviani G and Quaranta A A 1977 A review of some charge transport properties of silicon Solid-State Electron. 20 77-89

[17] Komma J, Schwarz C, Hofmann G, Heinert D and Nawrodt R 2012 Thermo-optic coefficient of silicon at $1550 \mathrm{~nm}$ and cryogenic temperatures Appl. Phys. Lett. 101041905

[18] Thompson J C and Younglove B A 1961 Thermal conductivity of silicon at low temperatures J. Phys. Chem. Solids 20 146-9

[19] Flubacher P, Leadbetter A J and Morrison J A 1959 The heat capacity of pure silicon and germanium and properties of their vibrational frequency spectra Phil. Mag. 4 273-94

[20] ET science team 2012 Einstein Gravitational Wave Telescope Conceptual Design Study code: ET0106C-10 (https://tds.ego-gw.it/ql/?c=7954)

[21] Nishi Y and Doering R 2012 Handbook of Semiconductor Manufacturing Technology 2nd edn (Boca Raton, FL: CRC Press)

[22] Cumming A V et al 2014 Silicon mirror suspensions for gravitational wave detectors Class. Quantum Grav. 31025017

[23] Harry G M et al 2007 Titania-doped tantala/silica coatings for gravitational-wave detection Class. Quantum Grav. 24405

[24] Cole G D, Zhang W, Martin M J, Ye J and Aspelmeyer M 2013 Tenfold reduction of Brownian noise in high-reflectivity optical coatings Nat. Photonics 7 644-50

[25] Abernathy M R et al 2011 Cryogenic mechanical loss measurements of heat-treated hafnium dioxide Class. Quantum Grav. 28195017 